\title{
The Development and Evaluation of Online Smoking Cessation Services: A Narrative Literature Review
}

\author{
Lewis Keane, ${ }^{1}$ Caroline Anderson, ${ }^{2}$ Donna Perez, ${ }^{2}$ and Becky Freeman ${ }^{1}$ \\ 1 Sydney School of Public Health, The University of Sydney, Sydney, NSW, Australia \\ 2 Screening and Prevention, Cancer Institute NSW, Eveleigh, Australia
}

\begin{abstract}
ntroduction: The use of quitlines for smoking cessation has contracted, with service providers adapting through the development of comprehensive interactive online smoking cessation services. The primary aim of this review is to investigate the continuum of online cessation services, innovations in design and service components, measures used in formative, process and outcome evaluations, as well as evidence of effectiveness.

Methods: This review includes the peer-reviewed literature, scholarly articles and the grey literature material. Databases searched included: PubMed, Google Scholar, SCOPUS and the Cochrane Collaboration.

Results/findings: 56 academic journal articles and the 5 grey literature reports met the inclusion criteria for this review. Developmental stages of online/combination services included: static websites, tailored feedback, email and text services, interactive components, social media, pharmacological offers; as well as social and professional support mechanisms. Innovations in online smoking cessation include: chat rooms, new recruitment strategies, mobile apps, service tailoring and messaging support groups. Online cessation services were significantly cheaper and more popular than quitlines; however, abstinence rates appear higher amongst quitline users.

Conclusions: Three likely catalysts for the shift from quitlines to online services are the rapid development of technology, increased internet access and the general movement of the goods and services sector to digital channels. The challenge for online cessation service providers is to leverage their comparative cost advantage and develop strategies that keep pace, engage users and increase service effectiveness.

Implications: Our paper synthesises a wide-range of the literature that evaluates the effectiveness and scope of online smoking cessation programs. Through applying this literature to the stages of evaluation framework, we also provide one of the first detailed roadmaps towards developing comprehensive evaluation methodology for online smoking cessation services.
\end{abstract}

\section{Introduction}

This past decade the goods and services sector has made a significant shift away from traditional face-to-face and telephone-based consumer interactions towards digital and technology-based forms of business and communication. This movement has had a strong effect on consumers, resulting in demand that all service providers, including the health sector, make use of accessible and familiar digital channels.

State and national smoking cessation service providers have borne witness to this effect. For example, calls to the New South Wales (NSW) Quitline in Australia have reduced by more than half from $2011(22,204)$ to 2016 $(10,746)$ (NSW Cancer Institute, 2015b). This declining trend in Quitline calls is not unique to NSW or Australia, with quitline usage in comparable settings reporting equally sharp declines. The North American Quitline Consortium reported longitudinal decreases in total direct callers, unique callers and service reach across U.S. States during the same time period (Rudie, 2016). Four likely catalysts for this change are the rapid development and reliance on new technologies, increasep

Address for correspondence: Lewis Keane, Level 6, The Hub - Charles Perkins Centre (D17) - The University of Sydney, NSW 2006, Australia. Email:lewis.keane@sydney.edu.au 
Table 1

The literature review questions and search terms

\begin{tabular}{|c|c|}
\hline Literature Review Questions & Search Terms \\
\hline $\begin{array}{l}\text { Part 1: a) What is the scale and scope of current online vs. telephone-based } \\
\text { or combination-style smoking cessation support services? }\end{array}$ & $\begin{array}{l}\text { (online OR internet) and (smoke* OR tobacco) and (service OR } \\
\text { program) and (cessation OR quit) }\end{array}$ \\
\hline b) What is the continuum of services available? & $\begin{array}{l}\text { (internet OR online OR telephone) and (quit smoke* OR cessation) } \\
\text { and (service or program* or intervention) }\end{array}$ \\
\hline $\begin{array}{l}\text { Part 2: What innovative features are being trialled in online smoking } \\
\text { cessation services? }\end{array}$ & $\begin{array}{l}\text { (Online OR internet Or mobile phone) AND (quit smoke* OR } \\
\text { cessation OR tobacco) AND (webchat OR app OR live chat OR } \\
\text { online chat OR e-chat) }\end{array}$ \\
\hline $\begin{array}{l}\text { Part } 3 \text { a) What measures and methods are used for assessing the } \\
\text { effectiveness of online and telephone-based smoking cessation services } \\
\text { and do these differ along the continuum of services? }\end{array}$ & $\begin{array}{l}\text { (Evaluation or monitor }{ }^{*} \text { or effect }{ }^{\star} \text { ) and (online or internet) and (quit } \\
\text { smoke* or tobacco) }^{*}\end{array}$ \\
\hline $\begin{array}{l}\text { b) What measures and methods would be recommended as suitable to } \\
\text { assess the effectiveness of online smoking cessation services? }\end{array}$ & $\begin{array}{l}\text { (Evaluation or monitor* or framework or effect* }{ }^{\star} \text { ) and (online or } \\
\text { internet) and (quit smoke* or tobacco) }\end{array}$ \\
\hline $\begin{array}{l}\text { Part 4: a) What evidence is there for the reach and effectiveness of online } \\
\text { smoking cessation support services? }\end{array}$ & $\begin{array}{l}\text { (Evaluation or effect*) and (online or internet) and (quit smoke* or } \\
\text { tobacco) }\end{array}$ \\
\hline $\begin{array}{l}\text { b) What evidence is there for the reach and effectiveness of } \\
\text { telephone-based smoking cessation support services? }\end{array}$ & (Quitline OR quit line) AND evaluate* AND (tobacco OR quit smoke*) \\
\hline
\end{tabular}

in social media use, market penetration of smart phones and wider Internet availability; all leading smokers to seek new online channels to aid in smoking cessation. These new channels are varied but include: phone applications (apps), mobile-based services, social media support, static informational websites, digital social support mechanisms and comprehensive interactive online cessation programs.

Interactive online cessation services have perhaps been the biggest development in response to falling quitline usage. In $201262 \%$ of U.S. states had developed some form of online self-directed or interactive service to aid smoking cessation, increasing to $76 \%$ in 2013 , and $96 \%$ in 2015 (Rudie, 2016). There is also strong evidence that when offered a choice of either a quitline, online service or a combination, the overwhelming majority select the online or combined service (An et al., 2010). This is also reflected in the Australian context with significant growth in the number of visits to the NSW Government's iCanQuit online service, increasing from 53,375 in 2011 to 851,376 in 2016 (NSW Cancer Institute, 2015a).

Despite this spike in popularity, the evidence base for the development and evaluation of online cessation services is limited. With significant investment in online services unmatched by strategic evidence to guide growth. This narrative literature review seeks to address this gap through the synthesis of a wide array of sources to provide a snapshot of the current development and evaluation stages of online smoking cessation services. The primary aim of this review is to investigate the scale and scope of current online, telephone-based, or combination-style smoking cessation services, and assess innovations in design and service components. Additionally, we map the current measures and methods used to evaluate online services throughout the formative, process and outcome evaluation stages and look at the current evidence of effectiveness.

\section{Methods}

As shown in (Table 1), four base subject questions for investigation were formulated to provide the structure and direction of the narrative literature review.

The following selection criteria were applied: (1) Language: the academic literature had to be published in the English language, (2) Time period: the academic literature had to be published between the 01/01/2006 and the 01/06/2016, (3) Relevance: the academic literature had to be relevant in answering one of more of the formulated literature review questions.

An extensive literature search was conducted sourcing relevant material from the peer-reviewed literature, scholarly article databases and the grey literature. Electronic databases PubMed, Google Scholar, SCOPUS and Cochrane Collaboration were included as sources of academic literature and scholarly articles. Additional, the grey literature material was sought throughout the search phase from non-academic sources including Centres for Disease Control (CDC) and Prevention, World Health Organisation, North American Quitline Consortium and abstracts from conferences.

\section{Results}

As shown in (Figure 1), a thorough search yielded a total of 3,072 peer-reviewed and scholarly articles, as well as the five grey literature reports. Of these, 1,953 duplicates were identified and removed, leaving 1,119 original articles for the screening phase of the review. LK screened 


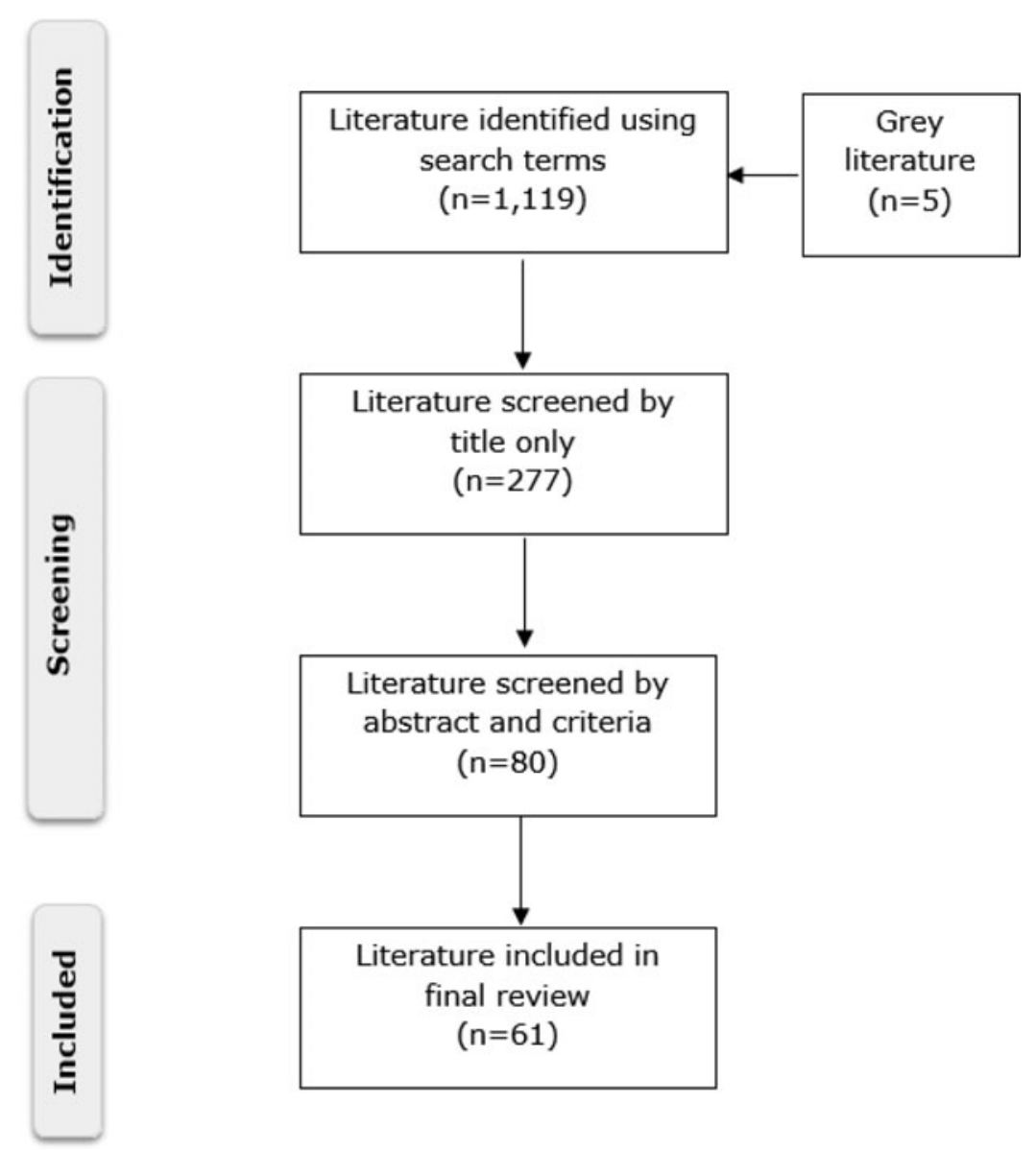

\section{Figure 1}

Search flow diagram.

these by title only, leaving 277 articles. LK and BF then screened the remaining articles by abstract, and applied the selection criteria, leaving 80 results. Remaining papers were screened by full-text, with conflicts addressed iteratively by $\mathrm{LK}$ and BF. Remaining conflicts were reviewed by DP and CA for inclusion or exclusion. 56 academic publications and five grey literature articles met the necessary criterion and were included in this review.

\section{Discussion}

Part 1: What Have Been the Development Stages of Online or Combination Style Smoking Cessation Services? What is the Continuum of Services Available?

During our search for this literature review, we found there is currently no single or core online smoking cessation treatment. Service providers offer an array of components both through, and alongside, online smoking cessation programs. Reasons for this appear varied but include funding restrictions, disparities in incidence and prevalence of tobacco use and differing cessation service development stages between regions.

Static tobacco cessation websites. As shown in (Figure 2), the first Internet tobacco services offered to smokers oc- curred after the move from information booklets to static websites. These websites were generally informational and non-tailored, containing content similar to a printed cessation booklet (Graham et al., 2016). Services classified as static websites can be both fully available at first viewing, or can deliver intervention components over time.

Tailored feedback. The next step in the continuum appeared to be the addition of tailored feedback. Tailored feedback consists of information provided to service users based on responses to one or more questionnaires. Tailoring of feedback was often based on user responses to a baseline assessment or on the basis of the user's current stage of quitting. The form of tailored messages and feedback has historically varied considerably from short messages based off quitting stage (Wangberg, Nilsen, Antypas, \& Gram, 2011), to several page reports based on personal demographics, as well as personal data input of updated smoking behaviour and relapse triggers (Etter, 2005).

Interactive online cessation programs. Interactive online cessation services evolved alongside increased access and use of the Internet. The scope of services offered is somewhat varied, but a majority seem to utilise a core group 


\section{Online smoking cessation}

\section{CONTINUUM}

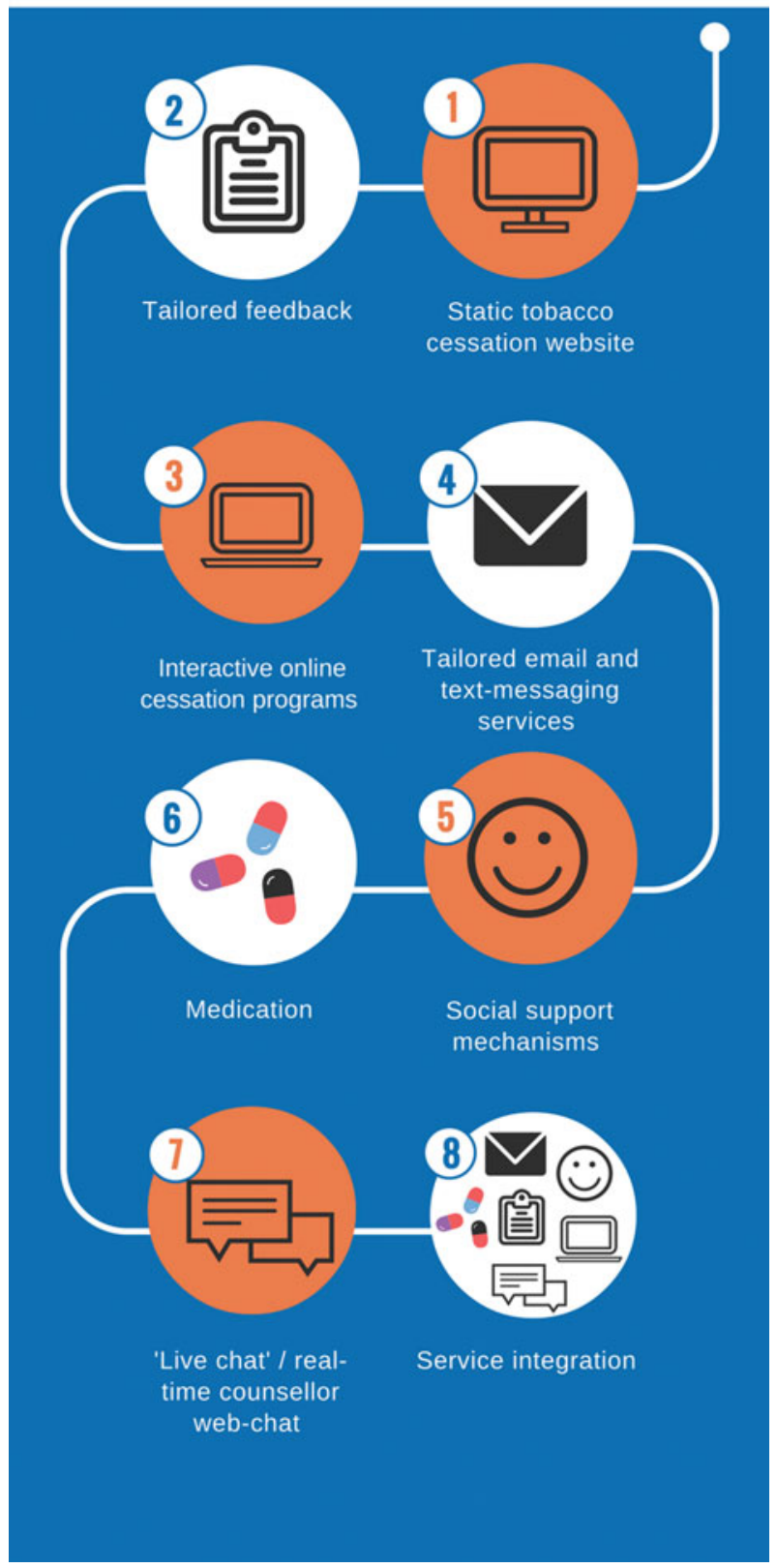

Figure 2

Development continuum infographic.

of components. An interactive tobacco tracker tracking cigarettes avoided, likely health improvements, progress updates, story sharing community features, cost savings calculator and personal quitting plan were the most widely used features overall (Heffner, Vilardaga, Mercer,
Kientz, \& Bricker, 2015; Nash, Vickerman, Kellogg, \& Zbikowski, 2015).

Tailored email and text-messaging services. Service providers have developed tailored email and textmessaging services alongside interactive cessation programs to further support service users. Tailored emails provided another avenue to reach and support smokers throughout the quitting process (Sadasivam, Allison, Ray, Ford, \& Houston, 2012), with behavioural change theory pointing to potential synergistic benefits of offering a range of service options (Webb, Joseph, Yardley, \& Michie, 2010). While text messages targeting a user's mobile phone or other electronic device help provide intensive support and leverage reminders in the initial stages of a user's quit attempt (Bock et al., 2013).

Social support mechanisms. The addition of social support features provided the next developmental stage. The most common form of social support was the provision of an asynchronous discussion forum where members could post questions, stories or experiences. This component represents a key social support mechanism whereby users receive feedback and support from other service users. Social Media and mobile phone apps were also developed to provide further means of social support during cessation attempts, to target younger smokers and increase service reach. The most popular amongst these are Facebook, as it has distinct strengths of rapid social support, user familiarity and convenience (Ramo, Liu, \& Prochaska, 2015).

Medications. Gaining high popularity in the U.S. providers also began offering free cessation medications by mail through online and quitline services (Rudie, 2016). Nicotine replacement therapy (NRT) patches, gum or lozenges were the most common forms of pharmacotherapy (Rudie, 2016), while other medications tested in clinical cessation trials included Varenicline and Bupropion (Graham et al., 2016). Rationale for this strategy appeared to be in providing further support to users, as well as attracting users to quitline and online services.

Live chat/real-time counselling services. Live chat or real-time online expert web chat counselling features were part of services offered by the U.K. National Health Service (NHS), U.S. National Cancer Institute, U.S. Department of Defence and the Government of Scotland. The U.S. National Cancer Institute integrated the live chat service into their national website in early 2015 (Keefe, 2015). They provide a form of immediate professional support in the face of decreasing quitline popularity.

Service integration. Integration of the above mentioned services is also becoming increasingly common. This is supported by behaviour change theory that smokers utilising a range of services are more likely to exhibit behaviour change than those relying on one form of cessation support (Webb et al., 2010). Integration involves 
either offering a choice of service, or options to utilise a combination of services when users first make contact with a state or national cessation service provider. A range of cross-sectional studies across the U.S. showed that when smokers were offered a choice via service integration, a large majority chose either the online option or a combined telephone online option (An et al., 2010; Nash et al., 2015).

\section{Part 2: What Components Show the Innovative Future of Smoking Cessation Services?}

Innovation in smoking cessation services has become commonplace and includes utilising a wide array of social media platforms and technology to develop supportive and interactive program components.

Strategic text-messaging services either in isolation, or alongside online services are becoming an integral part of smoking cessation programs. The benefits from text messaging interventions appear to be in reminding users as to why they had chosen to quit, reinforcing quit attempts in the short-term and reengaging users with services in the medium- to long-term (Haug, Schaub, Venzin, Meyer, \& John, 2013). They also seem most successful when used as part of an intensive short-term cessation program (Bock et al., 2013).

Facebook has also emerged as a multifaceted platform for smoking cessation service providers. Facebook has been trialled in a variety of ways including through a dedicated service provider page promoting recruitment, as a tool for monitoring groups of service users during evaluations, and as platform for social support (Cheung et al., 2015; Struik \& Baskerville, 2014). This social support feature, combined with the convenience offered by Facebook have been identified as distinct strengths, while managing privacy issues is a primary concern (Ramo et al., 2015).

Facebook apps are being continuously developed for smoking cessation, with the majority incorporating interactive, informational, and social features into their service. Exceptions to this were simple quit-date calculators and progress tracking apps (Jacobs, Cobb, Abroms, \& Graham, 2014). The majority of Facebook apps allowed posting to personal and friend's Facebook pages. This is another distinct advantage as this may lead to natural promotion of services to peer networks (Jacobs et al., 2014). Several of these Facebook apps also included community social support features to enable users to communicate with each other (Jacobs et al., 2014). In other realms of social media, Twitter groups also emerged as a possible inclusion as part of a short-term cessation intervention package (Pechmann, Delucchi, Lakon, \& Prochaska, 2016). Twitter groups were found to be engaging, low cost and scalable, making it a viable option as a cessation aid.

Novel social support strategies utilising chat rooms, WhatsApp and Facebook messenger groups were also found, with advantages identified as the ability to provide immediate support, cost effectiveness and ease of use (Cheung et al., 2015). Facebook discussion groups were also trialled with the addition of a professional moderator to promote discussion and provide feedback (Struik \& Baskerville, 2014). Evidence of effectiveness in aiding quitting was weak but indicated further research and testing was warranted.

Additionally, if social media is used as a tool for tracking participants and data collection during monitoring and evaluation, attrition rates from cessation studies and interventions could potentially be reduced. User emails are likely to change (e.g. change of work) and participants are easily lost to follow-up during studies. Facebook and other social media user accounts are less likely to change and may prove to be a better medium for communicating with, and tracking participants. They may also increase reengagement with users who relapse back to smoking.

With almost universal use of smart phones, mobile phone apps for smoking cessation are a commonplace tool (Bricker et al., 2014). In 2013, there were already over 400 smoking-related apps on the market (Bricker et al., 2014). The most used features of cessation apps were similar to those of online services and included a quit plan, tracking tools, progress tools and avenues for content sharing of cessation tips, coping with cravings and personal stories (Heffner et al., 2015). Adapting to the increasing popularity of cessation support apps, online cessation providers also routinely make services mobile optimised (adapted to be accessed easily via mobile phone), or in some cases created app versions of the services themselves.

'Live chat' or real-time in service web chat with cessation counsellors is in the early stages of use, with limited evaluations conducted into their cost effectiveness or impact on cessation outcomes. With quitline use declining globally, live chat can provide immediate professional support to users either planning a quit attempt, or throughout the quitting process. With the use of live chat services commonplace in a range of consumer and sales based industries, the movement into health-related support services is a natural progression. Distress help lines have successfully integrated live chat features to service clients with complex and serious mental health concerns (Haner \& Pepler, 2016).

\section{Part 3: What Measures and Methods are Used for Evaluating Online and Telephone-Based Smoking Cessation Services? What Measures and Methods Would be Recommended for Evaluating Online and Telephone-Based Smoking Cessation Services?}

Formative research. Best practice outlines that the primary focus of the formative evaluation stage should surround usability testing of new features, focus groups elucidating the most critical and engaging components of online services, as well as monitoring changes in technology access and behaviour of service users (Bauman \& Nutbeam, 2014). Although we found limited published formative evaluations, there was evidence of its use in investigating perceptions toward new and innovative 
features. This included studies into the likelihood of online users wanting the addition of Facebook as a quitting aid, as well as the typical user who would likely use the service (Ramo et al., 2015). This style of evaluation shouldn't just be confined to the planning stages of service implementation, but continually undertaken to ensure services shift with, and adapt to, the changing technological and tobacco policy landscape.

Process evaluation. There have been limited process evaluations published on online cessation programs. Despite this, a range of potential process indicators were extracted from various stages of evaluation included in this review.

Quantitative reach measures were reported by the $\mathrm{CDC}$ as a key component in Quitline evaluations, and could be transferrable to online service evaluations. The most common method for assessing reach was through the collection of demographic user data (Balmford, Borland, Li, \& Ferretter, 2009). This data assessed who the programs were reaching, how users were interacting with the service, exposure levels for user sub-groups, as well as the correlates between socioeconomic data, service use and cessation outcomes. Reporting shouldn't be confined to percentage use of the overall smoking population, but assess the ability of a service to target specific users including those in high-risk demographic groups, isolated users and underserved populations.

Associations between interactive component engagement by users, and positive improvements in smoking behaviour were assessed in several interventions (Strecher et al., 2008). As service components such as self-reporting of behaviour, identifying triggers, setting quit dates and tailored messaging grounded in behavioural change theory (Webb et al., 2010), evaluating user engagement with these features during process evaluations can be used in developing strategies to influence known early mediators of change.

These known early moderators and mediators of successful cessation fell into two main categories of either demographic or behavioural. For behavioural moderators, there was significant evidence pointing to self-efficacy, and the level of user willingness to quit, as the two primary mediators of successful cessation (Wangberg et al., 2011). Put simply, if a service user believes they can quit, and shows a desire to quit, this significantly increases their chances of successful smoking cessation. Demographics appear to be amongst the strongest mediators of engagement with cessation services. A recent systematic review of technology-based support via telephone or online for addiction found that 27 out of the 33 studies included in the review had a higher proportion of females than males engaged with services, ranging from $50.8 \%$ to $84 \%$ (Danielsson, Eriksson, \& Allebeck, 2014). The majority of online service users were younger, female and of higher socio-economic status than both the general smoking population and quitline users (Danielsson et al., 2014). However, evidence for demographic characteristics actu- ally leading to successful cessation was less clear-cut and largely inconclusive (Danielsson et al., 2014).

Although not always explicitly stated, evidence of the use of Fagerstrom indicators for nicotine dependence were found to be used as process indicators in several studies (Gainsbury \& Blaszczynski, 2011; Haug et al., 2013). Measures included time until first cigarette of the day, ability to refrain from smoking in forbidden areas, ability to forgo morning cigarettes, cigarettes smoked per day and if the user smokes when sick. The monitoring of longitudinal shifts in these indicators is crucial in evaluating if online services are positively influencing smoking behaviour change in online service users.

With the majority of online cessation services relying on the forum as their primary social support mechanism, there were relatively few studies examining their use or effectiveness. Online forum discourse analysis of posting behaviour was used to qualitatively assess the role of social support mechanisms in the cessation process. One novel method classified users as 'active' if they wrote posts and messages, 'passive' if they regularly viewed content without posting, or 'none' if they neither viewed nor added to discussion boards (Papandonatos, Erar, Stanton, \& Graham, 2016). A descriptive study was then used to assess key differences in users classified within the three groupings.

Continual monitoring of website performance, ease of use and speed with which the users become familiar with online services are also crucial inclusions during the process evaluation stage. Relevant evidence from Quitline evaluations show that user satisfaction of cessation services may act as a mediator for short-term abstinence (Jeong et al., 2012). Assessing indicators of perceived relevance and the ability to use services as intended can be superior to the evaluation of 'program satisfaction', which is subject to social desirability bias if asked during or immediately after program delivery (Bauman \& Nutbeam, 2014).

Outcome evaluation. The outcome evaluations of online smoking cessation services largely focus on measuring short-term or long-term outcome indicators.

For short-term outcome indicators, the monitoring and evaluation of quit tracking tool usage in online services are a particularly important inclusion in outcome evaluations (Heffner et al., 2015; Nash et al., 2015). Their use and the setting of cessation goals demonstrate a tangible intention to change smoking behaviour by a user. These tools are also important for smokers in the preparation stage of cessation as it offers information about getting ready for quitting, as well as tips and tricks for dealing with triggers and lapses.

Used scarcely throughout the literature but favoured from a behavioural change perspective is the monitoring and evaluation of quitlines and online services ability to promote quit attempts. This measure is an important inclusion as a short-term success indicator with evidence 
that a history of past quit attempts led to higher selfefficacy to quit than those without past attempts (Hyland et al., 2006).

For long-term outcome indicators, periodic point prevalence abstinence was the most used outcome measure (Bock et al., 2013; Danaher et al., 2015; Graham et al., 2015). This measure differed greatly in use across studies, ranging from 7 -day point prevalence abstinence at one-week follow-up, up to 30-day point prevalence abstinence at 13-month follow-up. Cost effectiveness analysis also warranted possible inclusion as an outcome evaluation variable (Graham et al., 2013). The methodology primarily used was to calculate cost of service per person quit, per quit attempt or per service user. In several studies, quitlines achieved significantly higher abstinence rates than online services (An et al., 2010; Neri et al., 2016). Despite this, when cost effectiveness indicators were included in outcome evaluations, online services show a considerable comparative advantage in achieving far lower cost per quit rate (Graham et al., 2013).

\section{Part 4: What Evidence is There For the Reach and Effectiveness} of Online Verses Telephone-Based Cessation Services?

Internationally, quitlines have seen a decrease in total direct callers, total unique callers and treatment reach (NSW Cancer Institute, 2015b; Rudie, 2016). In reaction to falling quitline usage and rise in the popularity of online services, developed nations in particular have begun to invest heavily in online service development.

The shift from quitlines to online cessation services was analysed across 10 U.S. state Quitlines that offered both services. Amongst all cessation service users, 79.9\% of participants had self-selected to utilise the combined quitline and online program (Nash et al., 2015). Demographic differences in typical users of each service, and between users who successfully and unsuccessfully utilised each service were also found. Those who chose online services were younger, healthier, more highly educated, non-ethnic minority and less likely to be highly nicotine-addicted.

While quitlines have been established as effective in aiding smokers to quit (Whittaker, McRobbie, Bullen, Rodgers, \& Gu, 2016), the effectiveness of online programmes is not as clear-cut. Evidence for the effectiveness of online services from meta-analyses were generally positive but far from homogenous. A range of systematic reviews published across the past seven years found online services to be superior to static information, untailored booklets, e-mail interventions and control groups. In some cases quitline services were significantly more effective than online services at promoting 30-day point prevalence abstinence in users (An et al., 2010). While in other cases there was no compelling evidence to support this claim. This lack of evidence, and disparity in agreement on quitline versus online effectiveness indicates that at the present stage of development, online programs are likely no more or less effective than other more intensive and expensive cessation services. This inconclusive agreement on relative effectiveness, combined with evidence that online services have the clear advantage of lower cost per quit and cost per user is a key leverage point for online services. With this cost advantage allowing increased investment in program effectiveness through other service additions. Examples of these strategies can be found all along the developmental continuum of online service components offered, as well as in the innovative nature of online service component development. A contemporary example of this innovation is the recent development of wearable cessation technology that monitor your daily heart rate and fitness, reporting how your vitals improve over time (Frederick, 2015).

\section{Conclusion}

This narrative literature review provides a critical overview of evidence relating to the development of online smoking cessation components, innovative and novel service additions, interventions and programs, monitoring and evaluation methodology, as well as evidence of reach and effectiveness of online smoking cessation services. Unique from the existing literature focusing on service effectiveness (Cheung, Wijnen, \& de Vries, 2017) or single component evaluation (Bock et al., 2013), our review synthesis evidence across the service life-course to inform development ranging from new features through to evaluation methodology. The movement of other common services online (banking, travel and ticket purchases), increased access to the internet, and advances in digital technology has likely influenced smokers to use these same channels to aid smoking cessation. Users expect cessation services to be available, engaging, effective and that they will be accessible through all channels. With consumers moving away from phone-based services and desktop computers, it is important that any changes developed place smartphone access as a priority. The primary areas for innovation in cessation support are the increased tailoring of support and feedback; strategic text-messaging services; online live chat and real-time counselling; and service integration. Facebook and mobile applications are increasingly common channels for expansion of services. However, service providers should also experiment with other channels such as Snapchat and Instagram, particularly for younger smokers. With the majority of online cessation services relying on web-based user forums as their primary social support mechanism, research is needed to guide and moderate these forums to improve service effectiveness.

Cessation services should continually improve evidence-based tools and interventions on offer to smokers, keeping pace with technology advances and changing media use. Service providers must also understand how to effectively promote these new services to maximise smoker recruitment. 
In conjunction with this drive for innovation, it is important to evaluate advances in support services. While online interactive smoking cessation service delivery has rapidly developed, there has not been a reciprocal rise in evaluations studying their effectiveness. We found a critical paucity of high-quality evaluation studies, most notably process evaluations, of online smoking cessation services. Without further studies elucidating the critical components and facilitators of successful cessation via increasingly popular online platforms, an exciting and crucial opportunity to assist tobacco users to quit could be compromised.

\section{Acknowledgements}

Katarzyna Bochynska - Cancer Institute NSW, MPH

Anita Dessaix - Cancer Institute NSW, MPH

We would like to acknowledge the insightful input of Katarzyna Bochynska and Anita Dessaix from the Cancer Institute NSW. As well as the Prevention Research Collaboration and Charles Perkins Centre at the University of Sydney.

\section{Sources of Funding}

The research which underpinned this narrative literature review was funded by the Cancer Institute NSW.

\section{Conflict of Interests}

None.

\section{Supplementary material}

To view supplementary material for this article, please visit https://doi.org/10.1017/jsc.2018.8

\section{References}

An, L. C., Betzner, A., Schillo, B., Luxenberg, M. G., Christenson, M., Wendling, A. et al. (2010). The comparative effectiveness of clinic, work-site, phone, and Web-based tobacco treatment programs. Nicotine \& Tobacco Research, 12(10), 989-996. doi:10.1093/ntr/ntq133.

Balmford, J., Borland, R., Li, L., \& Ferretter, I. (2009). Usage of an Internet smoking cessation resource: The Australian QuitCoach. Drug and Alcohol Review, 28(1), 66-72. doi:10.1111/j.1465-3362.2008.00009.x.

Bauman, A., \& Nutbeam, D. (2014). Evaluation in a Nutshell A practical guide to the evaluation of health promotion programs (Vol. 2nd Edition). Sydney, Australia: McGraw Hill Education.

Bock, B., Heron, K., Jennings, E., Morrow, K., Cobb, V., Magee, J. et al. (2013). A text message delivered smoking cessation intervention: The initial trial of TXT-2-Quit: Randomized controlled trial. JMIR Mhealth Uhealth, 1(2), e17. doi:10.2196/mhealth.2522.

Bricker, J. B., Mull, K. E., Kientz, J. A., Vilardaga, R., Mercer, L. D., Akioka, K. J. et al. (2014). Randomized, controlled pilot trial of a smartphone app for smoking cessation using acceptance and commitment therapy. Drug and Alcohol Dependence, 143, 87-94. doi:10.1016/j.drugalcdep.2014.07.006.

Cheung, Y. T., Chan, C. H., Lai, C. K., Chan, W. F., Wang, M. P., Li, H. C. et al. (2015). Using WhatsApp and Facebook online social groups for smoking relapse prevention for recent quitters: A pilot pragmatic cluster randomized controlled trial. Journal of Medical Internet Research, 17(10), e238. doi:10.2196/jmir.4829.

Cheung, Y. T., Wijnen, B., \& de Vries, H. (2017). A review of the theoretical basis, effects, and cost effectiveness of online smoking cessation interventions in the Netherlands: A mixed-methods approach. Journal of Medical Internet Research, 19(6), e230.

Danaher, B. G., Severson, H. H., Zhu, S.H., Andrews, J. A., Cummins, S. E., Lichtenstein, E. et al. (2015). Randomized controlled trial of the combined effects of web and quitline interventions for smokeless Tobacco cessation. Internet Interventions, 2(2), 143-151. doi:10.1016/j.invent.2015.02.005.

Danielsson, A. K., Eriksson, A. K., \& Allebeck, P. (2014). Technology-based support via telephone or web: A systematic review of the effects on smoking, alcohol use and gambling. Addictive Behaviors, 39(12), 1846-1868. doi:10.1016/j.addbeh.2014.06.007.

Etter, J. F. (2005). Comparing the efficacy of two internet-based, computer-tailored smoking cessation programs: A randomized trial. Journal of Medical Internet Research, 7(1), e2. doi:10.2196/jmir.7.1.e2.

Frederick, E. (2015). Using wearables for next-level aid in smoking cessation. Published in wearables.com, April 7th 2015, Available from: www.wearables.com/ wearables-to-quit-smoking-chono-therapeutics/.

Gainsbury, S., \& Blaszczynski, A. (2011). A Systematic review of internet-based therapy for the treatment of addictions. Clinical Psychology Review, 31(3), 490-498. doi:https://doi. org/10.1016/j.cpr.2010.11.007.

Graham, A. L., Carpenter, K. M., Cha, S., Cole, S., Jacobs, M. A., Raskob, M. et al. (2016). Systematic review and meta-analysis of internet interventions for smoking cessation among adults. Substance Abuse and Rehabilitation, 7, $55-69$.

Graham, A. L., Chang, Y., Fang, Y., Cobb, N. K., Tinkelman, D. S., Niaura, R. S. et al. (2013). Cost-effectiveness of internet and telephone treatment for smoking cessation: An economic evaluation of The iQUITT Study. Tobacco Control, 22(6), e11. doi:10.1136/tobaccocontrol-2012-050465.

Graham, A. L., Papandonatos, G. D., Cobb, C. O., Cobb, N. K., Niaura, R. S., Abrams, D. B. et al. (2015). Internet and telephone treatment for smoking cessation: Mediators and moderators of short-term abstinence. Nicotine \& Tobacco Research, 17(3), 299-308. doi:10.1093/ntr/ntu144.

Haner, D., \& Pepler, D. (2016). "Live Chat" clients at kids help phone: Individual characteristics and problem topics. Journal of the Canadian Academy of Child and Adolescent Psychiatry, 25(3), 138-144.

Haug, S., Schaub, M. P., Venzin, V., Meyer, C., \& John, U. (2013). Efficacy of a text message-based smoking cessation intervention for young people: A cluster randomized 
controlled trial. Journal of Medical Internet Research, 15(8), e171. doi:10.2196/jmir.2636.

Heffner, J. L., Vilardaga, R., Mercer, L. D., Kientz, J. A., \& Bricker, J. B. (2015). Feature-level analysis of a novel smartphone application for smoking cessation. American Journal of Drug and Alcohol Abuse, 41(1), 68-73. doi:10.3109/00952990.2014.977486.

Hyland, A., Borland, R., Li, Q., Yong, H. H., Mcneill, A., Fong, G., O'Connor, R. et al. (2006). Individual-level predictors of cessation behaviours among participants in the International Tobacco control (ITC) four country survey. Tobacco Control Journal, 15, iii83-iii94. doi:10.1136/tc.2005.013516.

Jacobs, M. A., Cobb, C. O., Abroms, L., \& Graham, A. L. (2014). Facebook apps for smoking cessation: A review of content and adherence to evidence-based guidelines. Journal of Medical Internet Research, 16(9), e205. doi:10.2196/jmir.3491.

Jeong, B. Y., Lim, M. K., Yun, E. H., Oh, J. K., Park, E. Y., Shin, S. H. et al. (2012). User satisfaction as a tool for assessment and improvement of quitline in the Republic of Korea. Nicotine \& Tobacco Research, 14(7), 816-823.

Keefe, B. (2015). Providing online live chat services for smokers looking to quit: Lessons learned from the integration of the national cancer institute's livehelp into smokefree. Gov Paper presented at the 2015 National Conference on Health Communication, Marketing and Media., Atlanta Georgia. https://cdc.confex.com/cdc/nphic15/webprogram/ Paper36561.html.

Nash, C. M., Vickerman, K. A., Kellogg, E. S., \& Zbikowski, S. M. (2015). Utilization of a web-based vs integrated phone/Web cessation program among 140,000 tobacco users: An evaluation across 10 free state quitlines. Journal of Medical Internet Research, 17(2), e36. doi:10.2196/jmir.3658.

Neri, A. J., Momin, B. R., Thompson, T. D., Kahende, J., Zhang, L., Puckett, M. C. et al. (2016). Use and effectiveness of quitlines versus Web-based tobacco cessation interventions among 4 state tobacco control programs. Cancer, 122(7), 1126-1133. doi:10.1002/cncr.29739.

NSW Cancer Institute. (2015a). 'iCanQuit' - online cessation service usage dataset. Cancer Institute New South Wales.

NSW Cancer Institute. (2015b). Usage of NSW Quitline dataset. Cancer Institute New South Wales.

Papandonatos, G. D., Erar, B., Stanton, C. A., \& Graham, A. L. (2016). Online community use predicts abstinence in combined internet/phone intervention for smoking cessation. Journal of Consulting and Clinical Psychology, doi:10.1037/ccp0000099.

Pechmann, C., Delucchi, K., Lakon, C. M., \& Prochaska, J. J. (2016). Randomised controlled trial evaluation of Tweet2Quit: A social network quit-smoking intervention. Tobacco Control. doi:10.1136/tobaccocontrol-2015-052768.

Ramo, D. E., Liu, H., \& Prochaska, J. J. (2015). A mixedmethods study of young adults' receptivity to using Facebook for smoking cessation: If you build it, will they come? American Journal of Health Promotion, 29(4), e126-e135. doi:10.4278/ajhp.130326-QUAL-128.

Rudie, M. (2016). Results from the FY2015 NAQC annual survey of Quitlines. Retrieved from http: //c.ymcdn.com/sites/www.naquitline.org/resource/resmgr/ 2015_Survey/finalweb2242016NAQCFY2015.pdf.

Sadasivam, R. S., Allison, J. J., Ray, M. N., Ford, D. E., \& Houston, T. K. (2012). Using a resource effect study pre-pilot to inform a large randomized trial: The Decide2Quit.Org Web-assisted tobacco intervention. AMIA Annual Symposium Proceedings, 2012, pp. 789-798.

Strecher, V. J., McClure, J., Alexander, G., Chakraborty, B., Nair, V., Konkel, J. et al. (2008). The role of engagement in a tailored web-based smoking cessation program: Randomized controlled trial. Journal of Medical Internet Research, 10(5), e36.

Struik, L. L., \& Baskerville, N. B. (2014). The role of facebook in crush the crave, a mobile- and social media-based smoking cessation intervention: Qualitative framework analysis of posts. Journal of Medical Internet Research, 16(7), e170. doi:10.2196/jmir.3189.

Wangberg, S. C., Nilsen, O., Antypas, K., \& Gram, I. T. (2011). Effect of tailoring in an internet-based intervention for smoking cessation: Randomized controlled trial. Journal of Medical Internet Research, 13(4), e121. doi:10.2196/jmir.1605.

Webb, L. T., Joseph, J., Yardley, L., \& Michie, S. (2010). Using the internet to promote health behavior change: A systematic review and meta-analysis of the impact of theoretical basis, use of behavior change techniques, and mode of delivery on efficacy. Journal of Medical Internet Research, 12(1), e4. doi:10.2196/jmir.1376.

Whittaker, R., McRobbie, H., Bullen, C., Rodgers, A., \& Gu, Y. (2016). Mobile phone-based interventions for smoking cessation. Cochrane Database of Systematic Reviews, 4, Cd006611. doi:10.1002/14651858.CD006611.pub4. 